VoL. 57 (1998) [55-58]

\title{
A NEW PROOF OF THE BANACH-STONE THEOREM
}

\author{
N.J. Cutland and G.B. Zimmer
}

\begin{abstract}
Banach's original proof of the Banach-Stone theorem for compact metric spaces uses peak functions, that is, continuous functions which assume their norm in just one point. We show by using nonstandard methods that the peak point approach also works for compact Hausdorff spaces. The peak functions are replaced by internal functions whose standard part is supported in one monad.
\end{abstract}

The Banach-Stone theorem still attracts a lot of attention; see [5] for a survey of the various generalisations. Banach's proof [1] of the Banach-Stone theorem for compact metric spaces uses peak functions. He characterises such functions in a way that is invariant under isometries. Eilenberg [4] gave a different intrinsic characterisation of peak functions and observed that Banach's proof can be carried out for completely regular, countably compact spaces satisfying the first countability axiom. For every point in $x \in X$ there is a function that peaks at $x$ if and only if $X$ is completely regular and every point of $X$ is a $G_{\delta}$. The proofs for compact Hausdorff spaces are either fairly involved or use the dual space of $C(X)$, the Riesz representation and extreme points (see $[2,3]$, or any other textbook on functional analysis) or they use maximal ideals. Our nonstandard proof for compact Hausdorff spaces replaces peak functions by internal functions whose standard part is supported in one monad.

Let $(X, \Omega)$ and $Y$ be compact Hausdorff spaces. For a point $x \in X$, the monad of $x$ is defined to be $\mu(x)=\bigcap\left\{{ }^{*} U: U\right.$ is a neighbourhood of $\left.x\right\}$. Here ${ }^{*} U$ denotes the nonstandard extension of the standard entity $U$.

The first Lemma goes back to Robinson [6]. It sums up the properties of the nonstandard extension of a compact Hausdorff space that we need:

LEMMA 1 . Let $X$ be a compact Hausdorff space. Then

(1) For $x, y \in X, \mu(x) \cap \mu(y) \neq \emptyset \Longleftrightarrow x=y$.

(2) For each $z \in{ }^{*} X, z \in \mu(x)$ for some $x \in X$.

By (1) and (2) the map ${ }^{\circ}:{ }^{*} X \rightarrow X$ which assigns the value $x$ to each $z \in \mu(x)$ is well defined.

Received 18th March, 1997

We thank Henry Cohen for suggesting this approach.

Copyright Clearance Centre, Inc. Serial-fee code: 0004-9729/98 SA2.00+0.00. 
THEOREM 2. Let $f \in{ }^{*} C(X)$ with $\|f\|_{\infty}=1$, where $X$ is a compact Hausdorff space. Then the following are equivalent:

(1) of has support in a single monad and does not change sign.

(2) For all $g \in C(X)$ with $\|g\|_{\infty}=1$

$$
\left\|f+\frac{1}{2} * g\right\|_{\infty}+\stackrel{\circ}{\circ} f-\frac{1}{2} * g \|_{\infty}=2 \text {. }
$$

Proof: Without loss of generality we may assume that $f\left(x_{1}\right)=1$. Set $x={ }^{\circ} x_{1}$. (1) $\Longrightarrow$ (2) follows from:

$$
\text { if } f \text { obeys (1) and }\|g\|_{\infty}=1 \text { then }\left\|f+\frac{1}{2}^{*} g\right\|_{\infty}=1+\frac{1}{2} g(x) \text {. }
$$

Applying (3) to $g$ and $-g$ yields (2).

To prove (3): ${ }^{\circ}\left\|f+(1 / 2)^{*} g\right\|_{\infty}=\max \left(9 f(z)+(1 / 2)^{*} g(z) \mid: z \in \epsilon^{*} X\right)$. Now for $z \not z x$, o $\left|f(z)+(1 / 2)^{*} g(z)\right|=(1 / 2) \circ g(z) \mid \leqslant(1 / 2) \leqslant 1+(1 / 2) g(x)$.

So

$$
\begin{aligned}
{ }^{\circ} \mid f+\frac{1}{2}^{*} g \|_{\infty} & =\max \left(\left|f(z)+\frac{1}{2}^{*} g(z)\right|: z \approx x\right) \\
& =\max \left({ }^{\circ} f(z): z \approx x\right)+\frac{1}{2} g(x) \\
& =1+\frac{1}{2} g(x) .
\end{aligned}
$$

$(2) \Longrightarrow(1):$ If $\delta=\min \left(\overline{{ }^{\circ} f(z): z \in^{*} X}\right)<0$, take $g \equiv 1$ and

$$
\text { 에 } f+\frac{1}{2}^{*} g \|_{\infty}=1+\frac{1}{2} \quad \text { and } \quad \text { o }\left\|f-\frac{1}{2}^{*} g\right\|_{\infty}=-\delta+\frac{1}{2} .
$$

Hence $\left\|f f+(1 / 2)^{*} g\right\|_{\infty}+q\left\|f-(1 / 2)^{*} g\right\|_{\infty}=2-\delta \neq 2$.

If ${ }^{\circ} f\left(x_{2}\right)=\delta>0$ with $x_{2} \not x_{1}$, take a continuous function $g: X \rightarrow[-1,1]$ with $g(x)=1$ and $g\left({ }^{\circ} x_{2}\right)=-1$. By Urysohn's Lemma, such a $g$ exists. Then

$$
\left\|f+\frac{1}{2}^{*} g\right\|_{\infty}=1+\frac{1}{2} \quad \text { and } \quad \text { o }\left\|f-\frac{1}{2}^{*} g\right\|_{\infty} \geqslant \delta+\frac{1}{2}
$$

Hence $q\left\|f+(1 / 2)^{*} g\right\|_{\infty}+0\left\|f-(1 / 2)^{*} g\right\|_{\infty} \geqslant 2+\delta>2$.

LEMma 3. Let $X$ be a compact Hausdorff space. For each $y \in{ }^{*} X$, there is a nonnegative function $f_{y} \in{ }^{*} C(X)$ with $\left\|f_{y}\right\|_{\infty}=1$, such that the support of ${ }^{\circ} f_{y}$ is 
contained in $\mu\left({ }^{\circ} y\right)$ and $f_{y}(y)=1$. This function can be chosen in a way that the family $\left\{f_{y}: y \in{ }^{*} X\right\}$ is internal.

Proof: There is a hyperfinite internal set $\mathcal{U}$ of subsets of ${ }^{*} X$ which contains the nonstandard extension of each open subset of $X$. For $y \in{ }^{*} X$, define a $*$-open set $V_{y} \subseteq \mu\left({ }^{\circ} y\right)$ by

$$
V_{y}=\bigcap(U: U \in \mathcal{U} \text { and } y \in U) .
$$

The internal version of Urysohn's Lemma applied to $y$ and ${ }^{*} X \backslash V_{y}$ produces a suitable function $f_{y}:{ }^{*} X \rightarrow{ }^{*}[0,1]$. Because the family of all sets $V_{y}$ is internal, the family of all functions $f_{y}$ is internal.

TheOREM 4. (Banach-Stone) Let $X$ and $Y$ be two compact Hausdorff spaces. If the spaces $C(X)$ and $C(Y)$ are isometrically isomporphic then the spaces $X$ and $Y$ are homeomorphic.

Proof: Let $T: C(X) \rightarrow C(Y)$ be an isometric isomorphism. For each $x \in X$, choose a function $f_{x} \in{ }^{*} C(X)$ as in Lemma 3. This function obeys condition (C) of Proposition 2. Condition (C) is invariant under isomorphisms, hence ${ }^{\circ} T\left(f_{x}\right)$ is also supported within one monad. Define $\varphi: X \rightarrow Y$ by

$$
\varphi(x)={ }^{\circ}\left(\operatorname{supp}^{\circ *} T\left(f_{x}\right)\right) .
$$

This is obviously a bijection. It remains to show that it is continuous. We do this by showing that $\varphi: X \rightarrow Y$ has an $S$-continuous lifting $\Phi:{ }^{*} X \rightarrow{ }^{*} Y$. For each $y \in{ }^{*} X$, choose an $f_{y}$ as in Lemma 3. Again, ${ }^{\circ} T\left(f_{y}\right)$ is supported within one monad and we may choose a point $\Phi(y) \in{ }^{*} Y$ for which $\left|{ }^{*} T\left(f_{y}\right)(\Phi(y))\right|=1$. Using the internal axiom of choice, we can do this for all points in ${ }^{*} X$ simultaneously. Here we use that the family of all $f_{y}$ for $y \in{ }^{*} X$ is internal. This defines an internal map $\Phi:{ }^{*} X \rightarrow{ }^{*} Y$.

If $y, z \in \mu(x)$, then $\left(f_{y}+f_{z}\right) /\left(\left\|f_{y}+f_{z}\right\|_{\infty}\right)$ does again satisfy condition $(C)$ and therefore the standard part of its image is supported within one monad, has norm one and does not change signs. Since the standard part of each of the three functions

$$
\frac{1}{\left\|f_{y}+f_{z}\right\|_{\infty}}{ }^{*} T\left(f_{y}\right), \quad \frac{1}{\left\|f_{y}+f_{z}\right\|_{\infty}}{ }^{*} T\left(f_{z}\right) \quad \text { and } \quad{ }^{*} T\left(\frac{f_{y}+f_{z}}{\left\|f_{y}+f_{z}\right\|_{\infty}}\right)
$$

is supported within one monad, all three must be supported in the same monad. This shows that $\Phi(y) \approx \Phi(z)$. Furthermore, it also shows that $\Phi(y)=\varphi(\xi)$.

\section{REFERENCES}

[1] S. Banach, Théorie des opérations lineaires (Chelsea, New York, 1932). 
[2] J.B. Conway, A course in functional analysis, Graduate Texts in Mathematics 96 (Springer-Verlag, Berlin, Heidelberg, New York, 1985).

[3] N. Dunford and J.T. Schwartz, Linear operators: General theory, Pure and Applied Mathematics 7 (Interscience, New York, 1958).

[4] S. Eilenberg, 'Banach space methods in Topology', Annals of Math. 43 (1942), 568-579.

[5] K. Jarosz, 'Isometries and small bound isomorphisms of function spaces', in Function spaces (Edwardsville, IL 1990), Lecture Notes in Pure and Appl. Math. 136 (Dekker, New York, 1992).

[6] A. Robinson, Non-standard analysis, (second revised edition) (North Holland, Amsterdam, 1974).

Department of Mathematics

University of Hull

Hull HU6 7RX

England
Division of Science and Mathematics

Mississippi University for Women

Columbus MS 39701

United States of America 